\title{
Die globale Bühne: Sportgroßereignisse im Spannungsfeld von politischer Inszenierung und demokratischen Reformimpulsen
}

Sport ist ein globales Massenphänomen, das mittlerweile im Alltagsleben zahlreicher Menschen einen festen Platz einnimmt. Sport dient dabei nicht nur der individuellen körperlichen Fitness oder dem mannschaftlichen Spiel und Kräftemessen, sondern er regt angesichts seines beträchtlichen Mobilisierungspotenzials auch zur gesellschaftlichen Kommunikation an (Bette 1990) und kann die unterschiedlichsten politischen und sozialen Prozesse befördern. Während Sport einerseits als Instrument von Integrationsprozessen (vgl. Coalter 2007; Braun/Nobis 2011), internationaler Verständigung (vgl. Armstrong/Giulianotti 1997; Sugden/Bairner 1999; Ehrhardt 2006) oder Entwicklungszusammenarbeit genutzt wird (vgl. Levermore 2008; Petry/Groll/Tokarski 2011), stellt er andererseits einen milliardenschweren Wirtschaftsfaktor dar, der auch Anlass zu Fehlentwicklungen wie Korruption oder Wettmanipulationen bietet (vgl. Kistner/Weinreich 1998; Hill 2008). Grundsätzlich ist zu konstatieren: Die fortschreitende Verflechtung des Sports mit anderen gesellschaftlichen Bereichen hat dazu geführt, dass es in den vergangenen Jahren einerseits zu einer immer stärkeren Instrumentalisierung des Sports für Interessen und Ziele jenseits des Sports gekommen ist, andererseits aber zugleich auch ein immer stärkeres Einwirken auf die Ausgestaltung des Sports und seiner Rahmenbedingungen zu verzeichnen ist (Mittag/Nieland 2007).

Besondere Bedeutung wird in diesem Zusammenhang Sportgroßereignissen zugeschrieben. Herausragende Sportgroßveranstaltungen wie Fußballweltmeisterschaften oder Olympische Spiele, aber auch die Tour de France oder Formel 1Rennen erzeugen beträchtliche, vor allem mediale Aufmerksamkeit und bieten damit zahlreichen Akteuren eine Projektionsfläche, auf der sie versuchen, ihre spezifischen Interessen zu realisieren. Während die Nutzung von Sportgroßereignissen für unmittelbare ökonomische Ziele und indirekte Wirkungen wie die Beeinflussung von Images in jüngster Zeit verstärkte Aufmerksamkeit gefunden hat (Kurscheidt 2007), sind die politischen Effekte von Sportgroßereignissen bislang weit weniger untersucht worden (Büch/Maenning/Schulke 2011). Die Beobachtung, dass autoritäre oder autokratische Regime Sportgroßveranstaltungen zur Selbstinszenierung und Machtstabilisierung nutzen, gehört zwar nicht zuletzt in Deutschland zu den 
Klassikern sportpolitischer Betrachtung (Oswald 2008; Emmerich 2011), die Potenziale von Sportgroßereignissen für Modernisierungs- und Demokratisierungsprozesse in autoritär regierten Staaten sind indes erst in jüngster Zeit verstärkt ins Blickfeld gerückt.

Vor dem Hintergrund dieser Debatten skizziert dieser Essay exemplarisch die Möglichkeiten, aber auch die Grenzen von Sportgroßereignissen für politische und gesellschaftliche Zielsetzungen in nicht-demokratisch regierten Staaten. Im Sinne der unterstellten Wechselwirkungen zwischen Sport und Politik wird auf der einen Seite gezeigt, inwieweit Sportgroßereignisse Impulse für politische Reformen und Modernisierungsprozesse liefern können, auf der anderen Seite aber auch erörtert, welche Ansätze verfolgt werden, um zu verhindern, dass Sportgroßveranstaltungen von Machthabern als globale Bühne für die eigene Machtsicherung inszeniert werden.

\section{Sportgroßereignisse: Definition, Rezeption und Vergabe}

Sportgroßereignisse können allgemein als zeitlich und räumlich begrenzte Vorgänge definiert werden, die durch ihren inhaltlichen Fokus und ihre öffentliche Aufmerksamkeit gekennzeichnet sind. Die in diesem Zusammenhang herangezogenen Kriterien und Indikatoren zur Klassifikation von Sportgroßereignissen weisen indes beträchtliche Unterschiede auf. So werden in einer häufig zitierten Studie von Hansruedi Müller und Jürg Stettler aus dem Jahr 1999 fünf Indikatoren angeführt, die vor allem auf quantitative Merkmale Bezug nehmen. Hierzu gehören die Anzahl der aktiven Sportler, der Betreuer und Funktionäre sowie der Zuschauer, das Veranstaltungsbudget und die mediale Verbreitung und Rezeption. In Verbindung mit der Ausdifferenzierung der Eventforschung sind diese Kategorien in der Folge erheblich ausgeweitet und verstärkt auf die mediale Rezeption bezogen worden. In modernen Gesellschaften sind Sportgroßereignisse wie Fußball-Weltmeisterschaften oder Olympische Spiele nicht nur sogenannte Mega-Events (Horne 2007), sondern immer auch Media-Events (Roche 2000). Letztere sind gemäß der verbreiteten Definition von Dayan und Katz (1992) als aus dem Alltag hervorstechende „Contests, Conquests, and Coronations“ zu verstehen. Tatsächlich generieren sportliche Großereignisse regelmäßig ein erhebliches und damit auch soziale, räumliche und ethnische Grenzen überschreitendes Zuschauerinteresse. So finden sich in der Liste der zehn meistgesehenen TV-Sendungen des Jahres 2010 in Deutschland ausschließlich Fußball-Übertragungen, davon auf den Plätzen eins bis neun Spiele der Fußball-Weltmeisterschaft in Südafrika (Zubayr/Gerhard 2011: 133). Die Übertragungen der WM-Spiele üben, wie Gerhard, Kessler und Gscheidle (2010: 383) in 
ihrer Analyse der Fernsehnutzung betonen, ,auf alle Altersklassen und die meisten Bevölkerungsgruppen eine große Attraktivität aus“. Gerade sportliche MediaEvents - Billings (2008) spricht von den Olympischen Spielen als ,biggest show on television" - sind in besonderer Weise geeignet, den seltenen Fall einer ungeteilten gesellschaftlichen Aufmerksamkeit und damit die Voraussetzung einer gesamtgesellschaftlichen Integration durch Medien herzustellen.

Im Gegensatz zur medialen Rezeption ist die Vergabe von Sportgroßereignissen ein wissenschaftlich bislang weitgehend unbeachtetes Sujet. Angesichts der zum Teil beträchtlichen Autonomie des Sports und des begrenzten Zugangs zu internen Entscheidungsprozessen von insbesondere internationalen Sportverbänden liegen hier bisher lediglich Medienberichte und vereinzelte Stellungnahmen von beteiligten Akteuren vor, jedoch keine systematischen Einblicke und Untersuchungen. Grundsätzlich kann zwischen drei - sich zum Teil überschneidenden - Typen der Veranstaltungsvergabe differenziert werden (Tröger 2011: 67). Häufig ist der Veranstalter ein Sportdachverband, der das Sportgroßereignis ausschreibt und dann an einen der Bewerber vergibt. Der Verband kann aber auch selbst als Ausrichter fungieren und sich dabei der Unterstützung anderer bedienen. Seltener kommt es vor, dass der Ausrichter das Sportgroßereignis selbst ausschreibt und Sportdachverbände lediglich eine Art Patronatsfunktion erfüllen.

Vor allem in den öffentlichkeitswirksamen Sportarten Fußball, Basketball, Handball und Eishockey erfolgt die Vergabe von Sportgroßereignissen bis heute zumeist durch ein Exekutivgremium, das auf der Grundlage eines entsprechenden - in der Verbandssatzung verankerten - Mandats die Entscheidung über die Austragungsstätte trifft. Diese Strukturen erscheinen vor allem in demokratietheoretischer Hinsicht problematisch, da die Vergabe eines Sportgroßereignisses angesichts der damit verbundenen Einnahmen vielfach die wichtigste Entscheidung eines Verbands darstellt, die jedoch nicht vom Kongress oder einem ähnlich bezeichneten Repräsentativorgan aller Verbandsmitglieder, sondern lediglich von einem kleinen Zirkel an der Verbandsspitze getroffen wird; die in politischen Systemen üblichen checks and balances fehlen hingegen in internationalen Sportverbänden. So fällt im Fall der FIFA nicht der Kongress, sondern das 24-köpfige Exekutivkomitee die Entscheidung über die Vergabe von Fußballweltmeisterschaften. Im Basketball erfolgt die Vergabe durch das 23-köpfige FIBA Central Board, im Handball durch den 17 Personen zählenden Rat (Council). Auch im Eishockey entscheidet ein 13-köpfiger Rat über die Vergabe der Weltmeisterschaft. Eine abweichende Regelung findet sich im Internationalen Olympischen Komitee: Hier erfolgt die Selektion der Städte, die sich überhaupt für die Olympischen Spiele bewerben können, zwar durch das Exekutivkomitee, die endgültige Entscheidung über den Ausrichter trifft dann aber die 
IOC-Session, das Parlament des Internationalen Olympischen Komitees, unter den Kandidaten.

Dass derartige Strukturen zu Problemen führen können, ist einer breiteren Öffentlichkeit im Zuge der Wiederwahl des FIFA-Präsidenten und der Vergabe der Weltmeisterschaften vor Auge geführt worden (vgl. Kistner 2012). Aufgrund von Korruptionsvorwürfen, -anklagen und nachgewiesenen Verfehlungen mussten zahlreiche Mitglieder des FIFA-Exekutivkomitees ihr Mandat niederlegen. Ob eher interne Reformprozesse der FIFA mit verschiedenen Reformkommissionen und dem Ansinnen, über die WM-Vergabe künftig den Kongress entscheiden zu lassen, oder aber externe Anstöße - wie die Überlegungen der Schweiz, Bestechung zum Straftatbestand zu machen und damit eine schärfere rechtliche Verfolgung von korrupten Funktionären zu ermöglichen - zu einer grundlegenden Änderung führen, bleibt abzuwarten.

Dass die Vergabe von Sportgroßereignissen in der letzten Dekade überhaupt zu verstärkter Aufmerksamkeit geführt hat, ist vor allem dem anhaltend wachsenden ökonomischen Potenzial des Sports zuzuschreiben. Während die Staaten der OECDWelt mittlerweile aber als weitgehend erschlossene bzw. gesättigte Sportmärkte gelten, wird den sogenannten BRICS-Staaten (Brasilien, Russland, Indien, China und Südafrika), aber auch weiteren Schwellenländern in Asien und Afrika, erhebliches Wachstumspotenzial zugeschrieben. Vor diesem Hintergrund kann es nicht überraschen, dass bei der Vergabe von Sportgroßereignissen vor allem Staaten berücksichtigt werden, deren Märkte noch sportökonomisches Wachstumspotenzial versprechen. Dass nicht wenige dieser wachstumsstarken Staaten (noch) keine stabilen Demokratien sind, ist die Kehrseite der Medaille. Die damit verbundenen Probleme rücken erst allmählich ins Bewusstsein der Funktionäre, die für humanitäre oder demokratiebezogene Überlegungen bislang eher begrenzte Sensibilität gezeigt haben.

\section{Das Beispiel Peking: Infrastruktureller Boom ohne Menschenrechtsverbesse- rungen}

Die olympischen Wettbewerbe in Peking 2008 rückten nicht nur aufgrund der wirtschaftlichen Dynamik des Landes in das Blickfeld sportpolitischer Betrachtung, sondern auch, weil erstmals seit 1936 wieder Spiele in einem nicht-demokratischen Staat stattfanden. Insbesondere die Menschenrechtsproblematik wurde sowohl von Befürwortern - in der Hoffnung auf mehr Menschenrechte durch Olympia - als auch von Gegnern - in der Erwartung verstärkter Repressionen - bei der Vergabe der Spiele an China als Argument ins Feld geführt. Daneben spielten aber auch ökono- 
mische Anreize eine Rolle. Offiziell betrug das Budget Pekings für die Spiele nur rund zwei Milliarden Dollar; da aber allein in Peking rund 40 Milliarden Dollar in Infrastrukturmaßnahmen und erhebliche Summen auch an anderen Wettkampforten investiert wurden, gelten die olympischen Spiele als wichtiger Impuls für Stadtentwicklung und Modernisierung. Ob Straßenerweiterungen oder neuangelegte Parks, zahlreiche Infrastrukturmaßnahmen der Hauptstadt wurden ebenso wie Maßnahmen zur Luftverbesserung erst im Zuge der Olympiaaktivitäten in Angriff genommen. Nicht selten wurde dabei aber auch auf das Mittel der Zwangsräumung für olympische Bauprojekte zurückgegriffen.

Die Erwartung, die sowohl von Experten (Pleiter 2008) als auch unter anderem in einer Umfrage von infratest dimap von 53\% der befragten Deutschen geäußert wurde, dass sich die Menschrechtssituation in China durch die Olympischen Spiele mittelfristig bessern wird, erfüllte sich jedoch nicht. Bereits im Vorfeld der Spiele wurden von den Behörden die Repressionen gegenüber Regimekritikern noch verstärkt, um einen Eindruck von Stabilität und Harmonie zu erwecken. Zahlreiche Oppositionelle wurden unter Hausarrest gestellt oder in Haft genommen, während zugleich die Medien streng zensiert wurden. Selbst die Kommunikation via Internet war während der Spiele nur eingeschränkt möglich. Lediglich Auslandsjournalisten konnten von behutsamen Liberalisierungstendenzen profitieren, die auch nach den Spielen anhielten.

Kritik erfuhr im Nachgang der Spiele insbesondere das IOC, aber auch Nationale Olympische Komitees und Politiker wurden ob ihrer Zurückhaltung gegenüber den anhaltenden Menschenrechtsverletzungen in China kritisiert. Da sich das IOC politischer Stellungnahmen weitgehend enthielt, wurde wiederholt angemahnt, nicht nur Korruptions- oder Umweltproblemen im Vorfeld von Olympischen Spielen durch einen Katalog von Standards Rechnung zu tragen, sondern künftig auch Menschenrechtsstandards stärker zu berücksichtigen. Die Vergabe der Winterspiele 2014 an Sotschi weist jedoch auf die Grenzen dieser Forderung hin.

\section{Die Beispiele Afrika-Cup und EURO 2012: Zwischen politischem Desinteresse und Protest}

Die beiden Kontinentalwettbewerbe Afrika-Cup und Europameisterschaft im Jahr 2012 stehen für die jüngsten Debatten um die Instrumentalisierung des Fußballs, aber auch für die Möglichkeiten und Grenzen des demokratischen Reformpotenzials des Fußballs. Äquatorialguinea, neben Gabun eines der beiden Ausrichterländer des Afrika-Cups, gilt nicht nur als diktatorisch regierter Staat, sondern auch als eines der korruptesten Länder der Welt. Trotz erheblicher Ölvorkommen und des höchs- 
ten Pro-Kopf-Einkommens Afrikas leiden weiten Teile der Bevölkerung unter Armut und Mangelerscheinungen. Bereits bei der Vergabe wurde dem afrikanischen Fußballverband CAF vorgehalten, mehr auf die zu erzielenden Einnahmen als auf die politischen Rahmenbedingungen geachtet zu haben. Machthaber Obiang nutzte den Wettbewerb zur Selbstdarstellung - so war auf den meisten Plakaten, die zum Turnier einluden, der Diktator abgebildet. Für jeden Vorrundensieg seiner Nationalmannschaft lobte Obiang eine Million Dollar für die Mannschaftskasse aus; die meisten Spieler der Startelf wurden zudem nicht in Äquatorialguinea geboren, sondern wurden, da man (erfolgreich) auf das Erreichen des Viertelfinales setzte, aus Spanien rekrutiert. Zu Protesten gegen die Verhältnisse im Land kam es im Vorfeld jedoch nur seitens von Nichtregierungsorganisationen. Neben den afrikanischen Fußballverbänden hielten sich auch die Politiker aus afrikanischen und europäischen Staaten auffällig zurück.

Kritischer und differenzierter präsentierte sich das Geschehen hingegen im Zuge der EURO 2012 in der Ukraine (und Polen). Als die Europameisterschaft 2007 vergeben wurde, hatten sich im Gefolge der „Orangenen Revolution“ zwar zunächst die westlich orientierten Kräfte um Wiktor Juschtschenko und Julija Tymoschenko durchgesetzt, sich aber nicht auf einen gemeinsamen Weg verständigen können, so dass es dem Kontrahenten Wiktor Janukowytsch letztlich gelang, die Präsidentschaftswahlen 2010 zu gewinnen und seine Macht auszubauen. Als Julija Timoschenko wegen Amtsmissbrauchs inhaftiert wurde und Vorwürfe publik wurden, die ehemalige Regierungschefin und Gegenkandidatin von Wiktor Janukowytsch sei in ihrer Gefängniszelle in Charkow misshandelt worden, setzte vor allem in Deutschland massive Kritik an der Austragung der Europameisterschaft in der Ukraine ein. Die Forderungen, namentlich aus den Reihen der Politik, reichten von Kartenrückgabe bis hin zu Boykottdrohungen. Allein die Fußballverbände, insbesondere die UEFA, hielten sich nicht nur mit Stellungnahmen zurück, UEFA-Präsident Michel Platini mahnte sogar Zurückhaltung an - konkret beim Kapitän der deutschen Nationalmannschaft. Wie differenziert sich das Geschehen aber darstellte, machte das Statement des neuen DFB-Präsidenten Wolfgang Niersbach deutlich, der von der Schweigelinie der UEFA abwich, als er einräumte, dass es humanitäre Probleme in der Ukraine gebe und es jedem freistehe, sich kritisch zu äußern. Eine Stellungnahme wie der viel zitierte Kommentar von Berti Vogts bei der Weltmeisterschaft 1978 in Argentinien, er habe ,,in der ganzen Zeit keinen einzigen politischen Gefangenen gesehen“, erscheint vor diesem Hintergrund kaum mehr denkbar.

Zahlreiche Politiker, darunter auch die gesamte EU-Kommission, bekundeten, nicht in die Ukraine reisen zu wollen; zu einem geschlossenen Politikerboykott der EU-Staaten kam es jedoch nicht. Seitens der teilnehmenden Mannschaften wurde 
ein Boykott nicht ernsthaft erwogen. Auffällig ist jedoch, dass im Fall der Ukraine entsprechende Aufrufe vor allem von denjenigen Politikern kamen, die sich im Wahlkampf befanden. Obgleich die ukrainische Regierung sich weitgehend unbeeindruckt zeigte, blieben die massiven Proteste von Politik und Medien nicht ohne Wirkung. Julija Tymoschenko erhielt medizinische Hilfe und Prozessbeobachter wurden bei der Überprüfung des Urteils gegen die ehemalige Regierungschefin zugelassen. Ob diese Zugeständnisse eher auf westliche Politikerproteste oder auf das Ansinnen des Präsidenten, die politischen und wirtschaftlichen Verhältnisse der Ukraine aus den Schlagzeilen zu bekommen, zurückzuführen sind, ist nicht unmittelbar messbar.

Auffällig ist indes die Zurückhaltung der internationalen Sportverbände, denen es an Sensibilität für die Menschenrechtsproblematik noch zu mangeln scheint. Während die Kontroversen über die Ukraine immer stärker schwelten, trafen zwei andere europäische Sportverbände ungerührt ähnliche Entscheidungen: Sowohl die Vergabe der Eishockey-Weltmeisterschaft 2014 in das diktatorisch regierte Weißrussland als auch die Vergabe der Basketball-Europameisterschaft 2015 unter zweifelhaften ökonomischen Rahmenbedingungen an die Ukraine zeugen davon, dass die zunehmende politische Sensibilität der Politik für Sportgroßereignisse bei den Verbänden noch nicht in gleichem Maße angekommen ist, die weiterhin auf ihre Autonomie und die unpolitische Dimension des Sports pochen.

\section{Herausforderungen für (Sport-)Politik, Sportverbände und Sportjournalisten}

Eine Geschichte der Olympischen Spiele ist auch immer eine der verschiedenen Boykotte und damit der Verlagerung des politischen Systemkampfes in den internationalen Sport. Nach dem Ende des Kalten Krieges schien die Bedeutung des Sports indes nicht nur für die Völkerverständigung, sondern auch für die Förderung der Demokratie zu wachsen. Dieser Eindruck erhielt mit den Spielen von Peking jedoch einen Dämpfer. Der Umgang des IOC, der Politik und der Medien mit der Menschenrechtsfrage offenbarte die Grenzen der Nutzung der Sportspiele zu politischen Zwecken.

Zwar haben sich in der internationalen Sportpolitik mittlerweile verstärkt Netzwerke gebildet (Groll/Gütt/Nölke 2010), die Fehlentwicklungen aufzeigen und kritische Nachfragen stellen. Die bisweilen fragwürdige Rolle der Verbände - gerade auf internationaler Ebene - ist dadurch jedoch nicht eingeschränkt worden. Wenn der Sport nicht nur zum Bestandteil von Friedens- und Entwicklungspolitik, sondern auch von demokratischen Entwicklungsoptionen werden will, dann muss die Sensibilität der Verbände bei der Vergabe von Sportgroßereignissen vertieft werden. 
Sportliche Großereignisse können eine Plattform für demokratische Reformen bieten - aber nur, wenn die Vergabepraxis hinterfragt wird, die (ökologische und infrastrukturelle) Nachhaltigkeit sichergestellt wird sowie Menschenrechtsfragen diskutiert werden.

Eine Patentlösung gibt es nicht, obwohl schon seit Jahren - verstärkt seit dem Sündenfall Atlanta (also der zunehmenden Kommerzialisierung der Spiele bei gleichzeitigen Korruptionserscheinungen) - Maßnahmen gefordert und vorgeschlagen werden. Sportgroßereignisse sind und bleiben von zentraler Bedeutung für demokratische Reformprozesse. Insbesondere vor dem Hintergrund der politischen Umwälzungen in zahlreichen Staaten der Welt, aber auch mit Blick auf die anhaltende Kritik an humanitären Problemen ist zu erwarten, dass die Verbände in $\mathrm{Zu}$ kunft stärkere Sensibilität dafür aufbringen, dass Sportereignisse nicht in einem politischen Vakuum stattfinden. Im Zeitalter der sozialen Netzwerke kann die Welt nicht mehr einfach mit dem Hinweis auf die vermeintlich unpolitische Dimension des Sports ausgeblendet werden. Infolgedessen ist auch die Art und Weise, wie wir über den Sport kommunizieren, wie wir Sport treiben und welchen Stellenwert wir dem Sport zugestehen, relevant. Zukünftig wird es verstärkt darum gehen, eine Balance zu finden zwischen den legitimen Interessen von Medien und Verbänden und ihrem ökonomischen Kalkül einerseits und einer fairen, kritischen und offenen Berichterstattung andererseits, die auch soziale und gesellschaftliche Aspekte berücksichtigt. Hier sind das IOC, die Medien und auch die Politik in Zusammenarbeit mit den nationalen und internationalen Sportverbänden - und vielleicht auch mit den Sportlern selbst - gefordert, nach Lösungen zu suchen.

\section{Literatur}

Armstrong, Gary/Giulianotti, Richard (Hrsg.), 1997: Entering the Field. New Perspectives in World Football, Oxford.

Bette, Karl-Heinrich, 1990: Sport als Thema geselliger Konversation. Zur Choreographie mikrosozialer Situationen, in: Willi Kleine/Wolfgang Fritsch (Hrsg.), Sport und Geselligkeit. Beiträge zu einer Theorie von Geselligkeit im Sport, Aachen, 61-80.

Billings, Andrew C., 2008: Olympic Media. Inside the biggest show on television, New York.

Braun, Sebastian/Nobis, Tina, 2011: Migration, Integration und Sport. Zivilgesellschaft vor Ort, Wiesbaden.

Büch, Martin-Peter/Maenning, Wolfgang/Schulke, Hans-Jürgen (Hrsg.), 2011: Internationale Sportevents im Umbruch?, Aachen. 
Coalter, Fred, 2007: A wider social role for sport: Who's keeping the score?, London/New York.

Dayan, Daniel/Katz, Elihu, 1992: Media Events. The Live Broadcasting of History, London.

Ehrhardt, Hans-Georg, 2006: Fußball und Völkerverständigung, in: Aus Politik und Zeitgeschichte (19), 18-25.

Emmerich, Alexander, 2011: Olympia 1936: Trügerischer Glanz eines mörderischen Systems, Köln.

Gerhard, Heinz/Kessler, Bernhard/Gscheidle, Claudia, 2010: Die Fußball-Weltmeisterschaft 2010 im Fernsehen, in: Media Perspektiven (9), 382-389.

Groll, Michael/Gütt, Matthias/Nölke, Andreas, 2010: Globale Sportpolitiknetzwerke, in: Walter Tokarski/Karen Petry (Hrsg.), Handbuch Sportpolitik, Schondorf, 142-157.

Hill, Declan, 2008: Sichere Siege, Köln.

Horne, John, 2007: The Four „Knowns” of Sports Mega-Events, in: Leisure Studies 26 (1), 81-96.

Kistner, Thomas, 2012: FIFA-Mafia. Die schmutzigen Geschäfte mit dem Weltfußball, München.

Kistner, Thomas/Weinreich, Jens, 1998: Das Milliardenspiel. Fußball, Geld und Medien, Frankfurt a. M.

Kurscheidt, Markus, 2007: Ökonomische Analyse von Sportgroßveranstaltungen: Ein integrierter Evaluierungs- und Management-Ansatz am Beispiel von Fußball-Weltmeisterschaften, Dissertation, Universität Paderborn.

Levermore, Roger, 2008: Sport: a new engine of development, in: Progress in Development Studies 8 (2), 183-190.

Mittag, Jürgen/Nieland, Jörg-Uwe (Hrsg.), 2007: Das Spiel mit dem Fußball. Interessen, Projektionen und Vereinnahmungen, Essen.

Müller, Hansruedi/Stettler, Jürg, 1999: Ökonomische Bedeutung sportlicher Grossveranstaltungen. Forschungsinstitut für Freizeit und Tourismus (FIF) der Universität Bern.

Oswald, Rudolf, 2008: „Fußball-Volksgemeinschaft“. Ideologie, Politik und Fanatismus im deutschen Fußball 1919-1964, Frankfurt a. M.

Petry, Karen/Groll, Michael/Tokarski, Walter (Hrsg.), 2011: Sport und internationale Entwicklungszusammenarbeit, Köln.

Pleiter, Dirk, 2008: Olympische Spiele 2008: Eine Chance für die Menschenrechte?, in: Mathias Klasberg/Kristin Kupfer (Hrsg.), Die Olympischen Sommerspiele in Peking 2008. Politik, Wirtschaft und Sport, Bochumer Jahrbuch zur Ostasienforschung (BJOAF) 31, 79-102. 
Roche, Maurice, 2000: Mega-Events and Media Culture. Sport and the Olympics, London/New York.

Sugden, John/Bairner, Alan, 1999: Sport in Divided Societies, Aachen.

Tröger, Walter, 2011: Vergabe von Sportveranstaltungen, in: Martin-Peter Büch/ Wolfgang Maenning/Hans-Jürgen Schulke (Hrsg.), Internationale Sportevents im Umbruch?, Aachen, 67-74.

Zubayr, Camille/Gerhard, Heinz, 2011: Tendenzen im Zuschauerverhalten. Fernsehgewohnheiten und Fernsehreichweiten im Jahr 2010, in: Media Perspektiven (3), 126-138.

Korrespondenzanschrift:

Univ.-Prof. Dr. Jürgen Mittag Deutsche Sporthochschule Köln Institut für Europäische Sportentwicklung und Freizeitforschung Jean Monnet Chair

Am Sportpark Müngersdorf 6 50933 Köln

E-Mail: mittag@dshs-koeln.de

Dr. Jörg-Uwe Nieland

Deutsche Sporthochschule Köln

Institut für Kommunikations- und Medienforschung

Aachener Str. 999 / Kirchweg 2

50933 Köln

E-Mail: J.Nieland@dshs-koeln.de 